\title{
A servo control system design using dynamic inversion ${ }^{\text {is }}$
}

\author{
Corrado Guarino Lo Bianco*, Aurelio Piazzi \\ Dip. di Ingegneria dell'Informazione, University of Parma, Parco Area delle Scienze, 181/A, 43100 Parma, Italy
}

Received 6 August 2001; accepted 18 January 2002

\begin{abstract}
This paper presents an inversion-based approach to the design of a dc motor-position servo system. Specifically, using the recently developed transition polynomials, a dynamic inversion procedure is established to determine a feedforward command signal to achieve high-performance position transfers. It is shown how to improve a traditional proportional and derivative controller feedback scheme and how a coordinated feedforward/feedback design using the new approach further improves the servo performances. Moreover, the methodology can easily comply with a voltage saturation avoidance constraint. Experimental results on a standard test bench highlight the effectiveness of the dynamic inversion idea. (C) 2002 Elsevier Science Ltd. All rights reserved.
\end{abstract}

Keywords: Servo design; Dynamic inversion; Saturation avoidance control; Transition polynomials; Optimal set-point transfers; de motors; PD controller

\section{Introduction}

Dynamic inversion is a feedforward methodology designed to achieve high performances in the control or regulation of dynamic systems. An ideal or desired output trajectory planning is set out and then, using the inverse system, the command input that will cause the desired output can be determined. Historically, dynamic inversion was first developed in the aerospace field where possible difficulties in implementing the designed command signals were overcome due to the great technological assets of the aerospace industry (Enns, Bugajski, Hendrick, \& Stein, 1994; Meyer, Hunt, \& Su, 1995).

Nowadays, the widespread availability of cheap microprocessors and related digital control technology makes dynamic inversion a methodology that can be profitably applied and developed for a great variety of control applications, starting from the very basic control problem of linear scalar set-point regulation. This line of control research has been recently

\footnotetext{
This work was partially supported by MURST scientific research funds under the framework of a COFIN 2000 project.

*Corresponding author. Tel.: +39-0521-905752; fax: +39-0521905723.

E-mail address: guarino@ce.unipr.it (C. Guarino Lo Bianco).
}

initiated in Piazzi and Visioli (2000, 2001a-c). For multivariable plants, the first result on set-point output transitions achieved with optimal inversionbased techniques is described in Perez and Devasia (2001).

In this paper, we develop a dynamic inversion approach to the design of a position servo system using a dc motor. Instrumental to this control design is the parameterized family of transition polynomials developed in Piazzi and Visioli (2001b) to shape "ideal" position transfers. An intrinsic advantage of the proposed method is the ease with which amplitude or rate limitations on the (voltage) manipulative input can be satisfied. Moreover, the minimum-phase property of the dc motor as a system plant makes the synthesis of the command signal a straightforward operation.

This paper is organized as follows. Section 2 shows how to improve an existing or classic servo control system using transition polynomials and inversion-based feedforward. Section 3 further improves the servo design by means of a combined feedforward/feedback design procedure. Experimental results are presented in Section 4 using standard laboratory equipments. An analysis of the performances against an artificially perturbed plant is also included. Section 5 reports concluding remarks. 


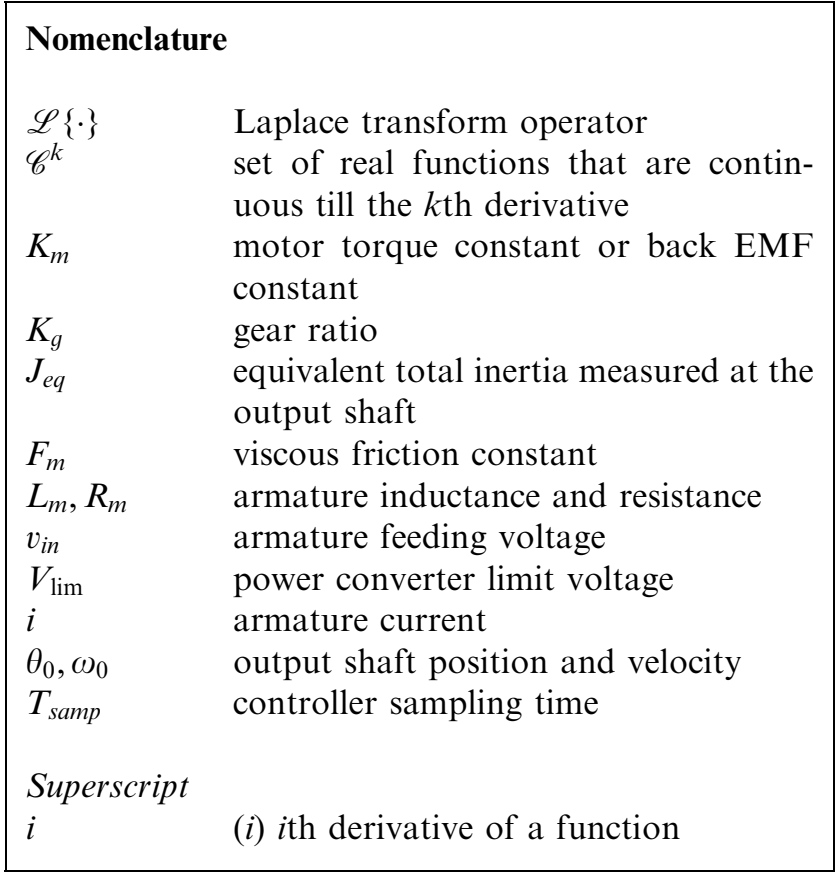

\section{Feedforward design via dynamic inversion}

Generally speaking, an effect position control for a servomechanism should satisfy the following requirements:

- fastest possible transient (minimum settling time);

- reduced overshoot (if possible, zero) to a step command;

- reduced steady-state error (if possible, zero);

- the controller output must never saturate.

The next subsection briefly describes a classic proportional and derivative (PD)-type feedback controller design. Section 2.2 shows how the servo performances can be improved by using the proposed inversion-based synthesis of the command input.

\subsection{The PD feedback controller}

The controlled plant is composed of a permanent magnet dc motor coupled to the output shaft by means of a reduction gear. Various approximations are usually made in order to design the controller. Firstly, the design is completely based on the nominal plant. Secondly, plant equations do not take into account various nonlinear effects introduced by friction (stiction, Stribeck effect, hysteresis, etc.) and backlash. Notwithstanding, such effects are particularly relevant in affecting the steady-state behavior especially when the velocity error constant of the control loop is not high (cf. Section 4). The design is then based on the reduction of the following classical dc motor model
(Kuo, 1995, p. 171):

$\frac{\mathrm{d} i}{\mathrm{~d} t}=-\frac{R_{m}}{L_{m}} i-\frac{K_{m} K_{g}}{L_{m}} \omega_{0}+\frac{v_{i n}}{L_{m}}$,

$\frac{\mathrm{d} \omega_{0}}{\mathrm{~d} t}=\frac{K_{m} K_{g}}{J_{e q}} i-\frac{F_{m}}{J_{e q}} \omega_{0}$,

$\frac{\mathrm{d} \theta_{0}}{\mathrm{~d} t}=\omega_{0}$.

The motor transfer function

$$
\begin{aligned}
& \frac{\Theta_{0}(s)}{v_{i n}(s)} \\
& =\frac{K_{m} K_{g}}{s\left[L_{m} J_{e q} s^{2}+\left(R_{m} J_{e q}+F_{m} L_{m}\right) s+\left(R_{m} F_{m}+K_{m}^{2} K_{g}^{2}\right)\right]}
\end{aligned}
$$

resulting from model (1) can be simplified by neglecting the fastest dynamic. By assuming that $L_{m}=0$, the following reduced transfer function is obtained:

$$
\begin{aligned}
G_{p}(s) & :=\frac{\Theta_{0}(s)}{v_{i n}(s)}=\frac{K_{m} K_{g}}{s\left[R_{m} J_{e q} s+R_{m} F_{m}+K_{m}^{2} K_{g}^{2}\right]} \\
& =\frac{1}{s(\alpha s+\beta)}
\end{aligned}
$$

where $\alpha=\left(R_{m} J_{e q} / K_{m} K_{g}\right.$ and $\left.\beta:=R_{m} F_{m}+K_{m}^{2} K_{g}^{2} / K_{m} K_{g}\right)$.

The position control system is schematically shown in Fig. 1. It uses a simple PD controller that will be digitally implemented. The zero-order hold can be taken into account by considering a delay time equal to $T_{\text {samp }} / 2$. Moreover, an additional delay of $T_{\text {samp }} / 2$ is added to consider also the latency delay caused by the discrete-time implementation of the controller. As usual, a first-order approximation may account for these delays so that the zero-order hold can be substituted by the transfer function:

$$
G_{s}(s):=\frac{1}{T_{\text {samp }} s+1} \text {. }
$$

The control scheme also evidences a feedback low-pass filter whose purpose is to cut off the noise deriving from the potentiometer used to detect the position of the output shaft. Its transfer function is

$$
G_{f}(s):=\frac{1}{\tau_{f} s+1} .
$$

In this control scheme, no current feedback is provided (i.e. the torque loop is missing) while a tachometric

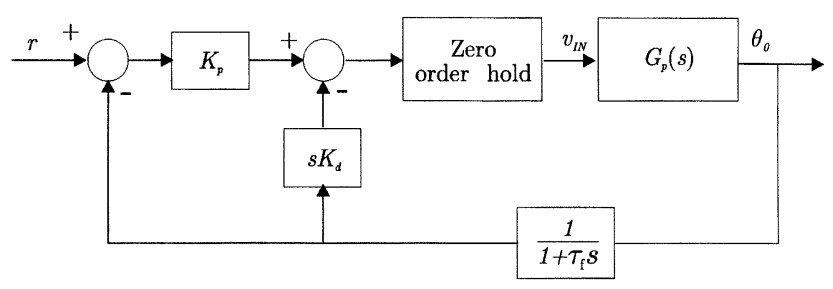

Fig. 1. Feedback control scheme. 
feedback is obtained through a discrete derivative of the position.

The proportional gain $K_{p}$ and the derivative gain $K_{d}$ are selected to assign the position of the closed-loop dominating poles. More precisely their damping ratio $\delta$ is directly assigned whereas their natural frequency $\omega_{n}$ can be indirectly evaluated by imposing the peak time $\left(t_{p}=\pi /\left(\omega_{n} \sqrt{1-\delta^{2}}\right)\right)$ of the closed-loop step response. The controller is tuned by selecting a combination of $\delta$ and $t_{p}$ in order to obtain the best-possible performances in response to a step-function reference. However, owing to the amplitude limitation on the maximum motor feeding voltage, a restriction on the feasible location of the dominant closed-loop poles emerges (cf. Section 4). Hence, a trade-off between overshoot and settling time arises and we note that to completely eliminate the output overshoot, the servo system exhibits an unacceptably large settling time.

\subsection{Command signal design via dynamic inversion}

The performances of the PD controller described in the previous subsection can be improved by synthesizing an appropriate command signal $r(t)$ for the closed-loop system of Fig. 1 to substitute the customary step command. This will determine a sharp reduction of both the overshoot and the settling time. Firstly, we choose the family of transition polynomials introduced in Piazzi and Visioli (2001b) as desired signals. Secondly, we solve the dynamic inversion problem by determining the command signal that causes the desired output. This approach has also been investigated in Piazzi and Visioli $(2001 \mathrm{a}, \mathrm{c})$ and it is briefly summarized herein.

Denote $r$ and $y$, respectively, as the input and the output signals of a proper minimum-phase linear plant defined by its transfer function:

$G(s):=\frac{b_{m} s^{m}+b_{m-1} s^{m-1}+\cdots+b_{0}}{s^{n}+a_{n-1} s^{n-1}+\cdots+a_{0}}$.

With the purpose of planning a smooth output transfer between two assigned output set-points (namely $y_{0}$ and $y_{1}$ ), we adopt the parameterized family of transition polynomials of Piazzi \& Visioli (2001b) to attain a desired motion without any oscillatory modes and with travelling time equal to the parameter $\tau$ :

$y(t ; \tau)$

$$
:= \begin{cases}y_{0}, & t<0, \\ y_{0}+\left(y_{1}-y_{0}\right) \frac{(2 k+1) !}{(k !)^{2} \tau^{2 k+1}} \int_{0}^{t} v^{k}(\tau-v)^{k} \mathrm{~d} v, & 0 \leqslant t \leqslant \tau, \\ y_{1}, & t>\tau\end{cases}
$$

The positive integer $k$ appearing in (5) can be freely chosen to ensure that $y(t) \in \mathscr{C}^{k}$ over $(-\infty,+\infty)$. The time derivative of (5) is given by

$y^{(1)}(t ; \tau)=\left(y_{1}-y_{0}\right) \frac{(2 k+1) !}{(k !)^{2} \tau^{2 k+1}} t^{k}(\tau-t)^{k}, \quad t \in[0, \tau]$.

The above expression (6) shows that $y(t ; \tau)$ is monotonically increasing or decreasing depending on the sign of $y_{1}-y_{0}$ exclusively. In particular, this guarantees that the planned output has no overshoot.

Relying on the stability of the zero dynamics of (4), the synthesis of the command signal is straightforward using

$R(s ; \tau)=G^{-1}(s) Y(s ; \tau)$,

where $Y(s ; \tau):=\mathscr{L}\{y(t ; \tau)\}$ and $R(s ; \tau):=\mathscr{L}\{r(t ; \tau)\}$; here, and in the following we set $y_{0}=0$ without any loss of generality. Using polynomial division rewrite $G^{-1}(s)$ as follows:

$G^{-1}(s)=\gamma_{\rho} s^{\rho}+\gamma_{\rho-1} s^{\rho-1}+\cdots+\gamma_{0}+H_{0}(s)$,

where $\rho:=n-m$ is the relative order (or relative degree) of $G(s)$ and $H_{0}(s)$ is the strictly proper stable transfer function representing the zero dynamics:

$H_{0}(s):=\frac{\delta_{m-1} s^{m-1}+\delta_{m-2} s^{m-2}+\cdots+\delta_{0}}{b_{m} s^{m}+b_{m-1} s^{m-1}+\cdots+b_{0}}$.

Define $\eta_{0}(t):=\mathscr{L}^{-1}\left\{H_{0}(s)\right\}$ and from (7) and immediately derive

$$
\begin{aligned}
r(t ; \tau)= & \gamma_{\rho} y^{(\rho)}(t ; \tau)+\gamma_{\rho-1} y^{(\rho-1)}(t ; \tau)+\ldots \\
& +\gamma_{0} y(t ; \tau)+\int_{0}^{t} \eta_{0}(t-v) y(v ; \tau) \mathrm{d} v, \quad t \geqslant 0 .
\end{aligned}
$$

It is known (Polderman \& Willems, 1998, p. 112; Piazzi \& Visioli, 2001b) that the command signal $r(t)$ is of class $\mathscr{C}^{l}$ if and only if $y(t)$ is of class $\mathscr{C}^{l+\rho}$. Hence, the appropriate choice of $k$ in (5) makes it possible to obtain an arbitrarily smooth command signal.

For the servo system considered in this paper, we have set $y \equiv \theta_{0}$ and introduced the transfer function of the closed-loop control scheme of Fig. 1 as

$$
\begin{aligned}
G_{o}(s) & :=\frac{\Theta_{o}(s)}{R(s)} \\
& =\frac{K_{p}\left(1+\tau_{f} s\right)}{s\left(1+\tau_{f} s\right)\left(1+\tau_{s} s\right)(\alpha s+\beta)+s K_{d}+K_{p}} .
\end{aligned}
$$

This $G_{o}(s)$ is supposed to be stable owing to the controller synthesis proposed in the previous subsection and has relative order $\rho=3$. By means of the polynomial division, it is possible to obtain the $\gamma_{i}$ parameters and the explicit expression of $\eta_{0}(t)$ :

$$
\begin{aligned}
& \gamma_{3}=\frac{\tau_{s} \alpha}{K_{p}}, \quad \gamma_{2}=\frac{\tau_{s} \beta+\alpha}{K_{p}}, \quad \gamma_{1}=\frac{\beta}{K_{p}}, \quad \gamma_{0}=\frac{K_{d}}{\tau_{f} K_{p}}, \\
& \eta_{0}(t)=\left(1-\frac{K_{d}}{\tau_{f} K_{p}}\right) \frac{\mathrm{e}^{-t / \tau_{f}}}{\tau_{f}} .
\end{aligned}
$$


The system command signal can be thus explicitly given by

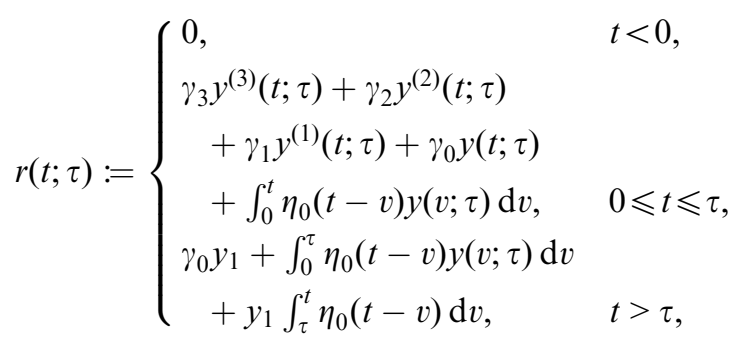

where, having chosen $k=3$ to obtain a continuous command signal, for $t \in[0, \tau]$

$y(t ; \tau)=y_{0}+140 \frac{y_{1}}{\tau^{7}}\left(-\frac{t^{7}}{7}+\frac{\tau t^{6}}{2}-\frac{3 \tau^{2} t^{5}}{5}+\frac{\tau^{3} t^{4}}{4}\right)$

$y^{(1)}(t ; \tau)=140 \frac{y_{1}}{\tau^{7}}\left(-t^{6}+3 \tau t^{5}-3 \tau^{2} t^{4}+\tau^{3} t^{3}\right)$,

$y^{(2)}(t ; \tau)=420 \frac{y_{1}}{\tau^{7}}\left(-2 t^{5}+5 \tau t^{4}-4 \tau^{2} t^{3}+\tau^{3} t^{2}\right)$,

$y^{(3)}(t ; \tau)=840 \frac{y_{1}}{\tau^{7}}\left(-5 t^{4}+10 \tau t^{3}-6 \tau^{2} t^{2}+\tau^{3} t\right)$.

One degree of freedom still remains for the complete definition of the command signal $r(t ; \tau)$ : the transient time $\tau$. An obvious choice is to minimize $\tau$ to get the fastest possible transient. This means finding the minimum $\tau$ under the constraint of saturation avoidance for the motor feeding voltage. To this end let us denote with $v_{i n}(t ; \tau)$ the motor voltage input caused by the command input $r(t ; \tau)$. Hence the following semi-infinite generalized optimization problem is considered:

$\tau^{*}=\arg \left\{\min \tau:\left|v_{\text {in }}(t ; \tau)\right| \leqslant V_{\lim }, \forall t \in[0, \tau]\right\}$,

where $V_{\lim }$ is the power converter saturation voltage. It is worth noting that function $v_{i n}(t ; \tau)$ does not depend on the controller parameters but it is exclusively linked to the motor transfer function $G_{p}(s)$ (3) through the relation:

$v_{\text {in }}(t ; \tau)=\mathscr{L}^{-1}\left[G_{p}^{-1}(s) Y(s ; \tau)\right]$.

Hence, applying the dynamic inversion procedure delineated in (7)-(10) and taking into account the structure of $G_{p}(s)$ given by (3), we obtain

$v_{\text {in }}(t ; \tau)=\gamma_{p 2} y^{(2)}(t ; \tau)+\gamma_{p 1} y^{(1)}(t ; \tau)$,

where $\gamma_{p 1}$ and $\gamma_{p 2}$ are appropriate real coefficients.

Problem (17) can be solved using specific algorithms for semi-infinite optimization (Teo, Rehbock, \& Jennings, 1993; Guarino Lo Bianco \& Piazzi, 2001a, $2001 \mathrm{~b})$. However, for the case of $y(t ; \tau) \in \mathscr{C}^{3}$, it is possible to equivalently reformulate problem (17) to obtain a standard finite optimization problem. Indeed, with the help of tools for symbolic manipulation, the five possible relative extrema of $v_{i n}(t ; \tau)$ over the domain $[0, \tau]$ can be expressed in closed form; we denote them with $\hat{v}_{i}(\tau)$, $i=1, \ldots, 5$. Therefore, problem (17) is equivalent to

$\tau^{*}=\arg \left\{\min \tau:\left|\hat{v}_{i}(\tau)\right| \leqslant V_{\lim }, i=1,2, \ldots, 5\right\}$

and can be solved with standard nonlinear programming tools or with a more straightforward bisectiontype algorithm (Piazzi \& Visioli, 2001b).

The previous considerations make it evident that $\tau^{*}$ depends only on the plant transfer function and on the profile chosen for the output plant. This is an interesting feature of the proposed dynamic inversion approach. On the contrary, adopting a purely feedback control scheme with a traditional step command, the avoidance of the voltage saturation should be sought by imposing constraints on the controller parameters.

\section{Coordinated feedforward/feedback design using dynamic inversion}

In the following it will be supposed, in agreement with adopted inversion-based approach, that the output signal has already been fixed as $y\left(t ; \tau^{*}\right)$, the optimal transfer defined by (17) and (19). Hence, the insertion of a feedback controller is justified in order to reduce the sensitivity of the servo system to variations in the motor plant parameters and to unmodelled dynamics (such as friction and other nonlinear phenomena) (Horowitz, 1963, p. 94). As a consequence, the controller design should follow these guidelines: (a) closed-loop stability is attained, (b) the controller bandwidth is as large as possible, and (c) the loop velocity error constant $K_{v}$ is as high as possible.

Using these guidelines it will be possible to greatly reduce, as noted in Piazzi and Visioli (2001a), the effects of the plant uncertainties over the range of frequencies of the command signal. It is worth noting that a controller designed according to the proposed approach will be quite different from a controller synthesized according to the traditional setup with step-command signals. In particular, the new approach makes it possible to obtain high values for $K_{v}$ with respect to that of a traditional controller. For the case at hand, an appropriate control scheme of the servo system is shown in Fig. 2. The fixed-structure controller $G_{c}(s)$ is chosen to cancel the two "slowest" plant poles and to have a pair of complex poles according to a Butterworth configuration:

$G_{c}(s):=K_{c} \frac{(1+\lambda s)\left(1+\tau_{s} s\right)}{1+\sqrt{2} s / \omega_{c}+s^{2} / \omega_{c}^{2}}$,

where $\lambda:=\alpha / \beta$ is the plant time-constant dominating pole and $\tau_{s}$ is the equivalent time constant of the zeroorder hold. The controller design parameters are then $K_{c}$, the controller static gain that is proportional to the velocity error constant $K_{v}$, and $\omega_{c}$, the Butterworth natural frequency of the controller poles. 


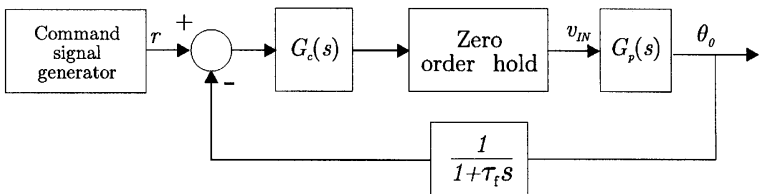

Fig. 2. The new feedback control scheme.

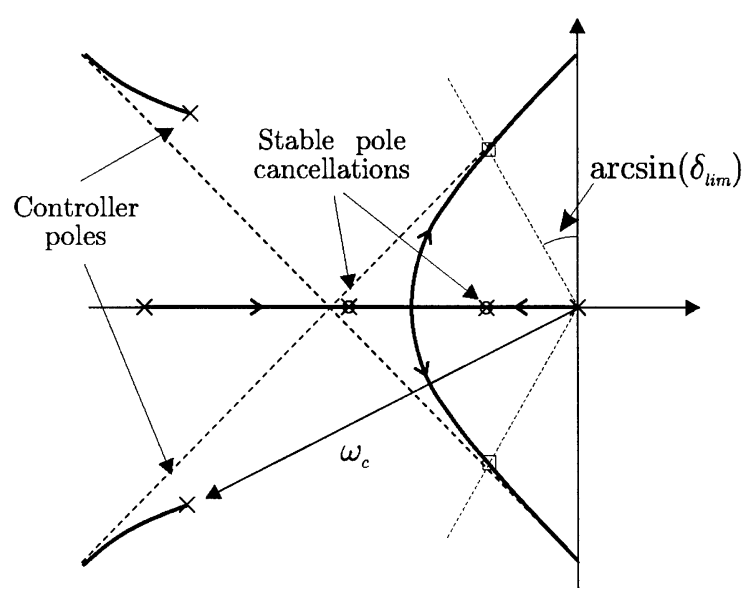

Fig. 3. Schematic root locus; the squares denote the closed-loop dominant poles.

In accordance with the above, design guidelines proceed as follows:

(1) Firstly, choose the highest $\omega_{c}$ value compatible with the digital implementation and in any case the factor $\omega_{c} \lambda$ should not be too high (in general, the time constants of the controller should not be too small compared with those of the plant).

(2) Secondly, assign the minimum damping ratio $\delta_{\lim }$ for the closed-loop dominant poles and then choose the maximal $K_{c}$ for which the corresponding dominant poles have a damping ratio greater or equal to the assigned $\delta_{\lim }$.

The choice of the maximal $K_{c}$ is illustrated in Fig. 3 showing a schematic root locus. We remark that the assigned damping ratio $\delta_{\lim }$ can be chosen to be much smaller than the usual values related to a traditional controller design because, having adopted the dynamic inversion approach, the actual over-shooting of the setpoint transfer has no relation to the closed-loop dominant poles.

The final design step is the determination of the command signal $r\left(t ; \tau^{*}\right)$ through dynamic inversion. Considering that the pertinent closed-loop function is

$$
\begin{aligned}
G_{0}(s) & :=\frac{\Theta_{0}(s)}{R(s)} \\
& =\frac{K_{c}\left(1+\tau_{f} s\right)}{\beta s\left(1+\tau_{f} s\right)\left(1+\sqrt{2}\left(s / \omega_{c}\right)+s^{2} / \omega_{c}^{2}\right)+K_{c}},
\end{aligned}
$$

then we obtain the feedforward command signal as $r\left(t ; \tau^{*}\right)=\mathscr{L}^{-1}\left[G_{o}^{-1}(s) Y\left(s ; \tau^{*}\right)\right]$.

According to the dynamic inversion procedure described in (8)-(10) we explicitly derive the command signal as

$$
\begin{aligned}
r\left(t ; \tau^{*}\right)= & \frac{\beta}{\omega_{c}^{2} K_{c}} y^{(3)}\left(t ; \tau^{*}\right)+\frac{\beta \sqrt{2}}{\omega_{c} K_{c}} y^{(2)}\left(t ; \tau^{*}\right)+\frac{\beta}{K_{c}} y^{(1)}\left(t ; \tau^{*}\right) \\
& +\frac{1}{\tau_{f}} \int_{0}^{t} \mathrm{e}^{-(t-v) / \tau_{f}} y\left(v ; \tau^{*}\right) \mathrm{d} v .
\end{aligned}
$$

\section{Experimental results}

To validate the approach proposed in this paper, we used the experimental setup developed by Quanser Consulting. The test bench is based on a personal computer controlling, by means of a built in $\mathrm{I} / \mathrm{O}$ board and an external power converter, a dc servo motor (see Fig. 4). The personal computer acts as a rapid prototyping station: the controller is introduced as a Simulink scheme and converted by the Real Time Workshop package into an actual digital controller. For all the experiments, the sampling time $\left(T_{\text {samp }}\right)$ is $5 \times 10^{-3} \mathrm{~s}$ and the cut-off frequency of the input filtering is $25 \mathrm{~Hz}$ (corresponding to $\tau_{f}=6.37 \times 10^{-3} \mathrm{~s}$ ). Continuous time controllers have been automatically discretized, using the Runge-Kutta solver, by the Real Time Workshop to obtain equivalent discrete time implementations.

The nominal plant coefficients used for the controller design are listed in Table 1. They are derived from the Quanser Handbook with the sole exception of the friction coefficient $F_{m}$ which was not available and which has been experimentally estimated. The three plant poles, evaluated from the parameters listed in Table 1 , are located at $0,-61.84$ and $-14387.47 \mathrm{~s}^{-1}$. According to the simplification proposed in Section 2.1, the fastest plant pole has been neglected by posing $L_{m}=0$. The resulting transfer function has two poles

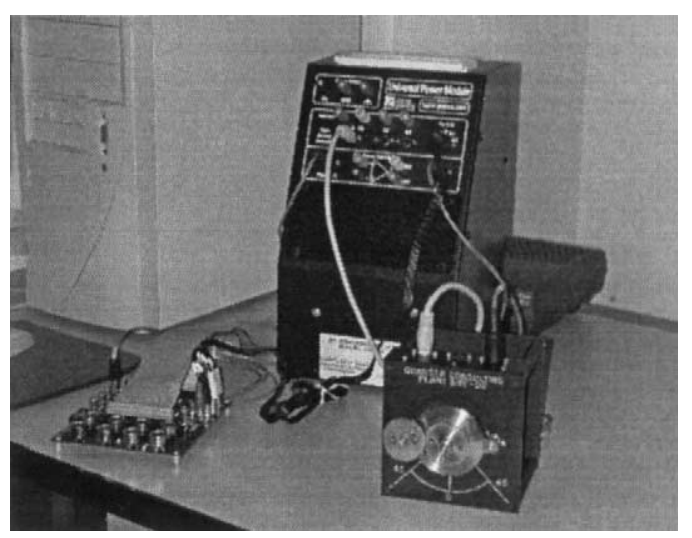

Fig. 4. The experimental test bench at the Automatica lab of the University of Parma. 
Table 1

DC motor and load parameters

$K_{m}=7.67 \times 10^{-3} \mathrm{Nm} / \mathrm{A}$ or $\mathrm{V} /(\mathrm{rad} / \mathrm{s})$

$K_{g}=70$

$J_{e q}=0.195 \times 10^{-2} \mathrm{~kg} \mathrm{~m}^{2}$

$F_{m}=0.95 \times 10^{-2} \mathrm{~N} \mathrm{~m} \mathrm{~s}$

$L_{m}=0.18 \times 10^{-3} \mathrm{H}$

$R_{m}=2.6 \Omega$

$V_{\lim }=5 \mathrm{~V}$

placed, respectively, at 0 and $-61.60 \mathrm{~s}^{-1}$ and a plant velocity constant $\beta^{-1}=1.72 \mathrm{~s}^{-1}$ (cf. (2) and (3)).

In the following, the control strategies presented in the previous sections are verified and compared on the actual plant. The robustness of the achieved performances is discussed in Section 4.3 where further experiments on a perturbed plant are reported.

\subsection{Experimental results with the standard PD controller synthesis}

During this first stage the dynamic inversion approach is not used. Consider the simple controller of Fig. 1 and optimally tune its parameters in accordance with the procedure proposed in Section 2. Choose $y_{0}=$ $0^{\circ}$ and $y_{1}=45^{\circ}$. Several values of $\delta \in[0.7,1)$ have been tested and, for each of them, the minimum $t_{p}$ compatible with the limit on the maximum voltage has been selected. Hence, the PD controller used for the experiments has been synthesized on the basis of the best compromise between overshoot, settling time and steady-state error. Its resulting parameters are $K_{p}=6.234 \mathrm{~V} / \mathrm{rad}$ and $K_{d}=-0.1190 \mathrm{~V} \mathrm{~s} / \mathrm{rad}$. Consequently, the dominating poles of the closed-loop system have a damping ratio $\delta=0.95$ and the loop velocity error constant is $K_{v}=15.73 \mathrm{~s}^{-1}$. The resulting step response of the closed-loop system is shown in Fig. 5. By defining the settling time $t_{s}$ as the minimum time for which the output definitely belongs to the $\pm 2 \%$ range of the steady-state value, it results that $t_{s}=0.3103 \mathrm{~s}$. The experimental experience indicates that a large proportional gain $K_{p}$ has to be chosen in order to achieve fast transients, to increase the velocity constant $K_{v}$ and to reduce the steady-state error. Unfortunately, a value of $K_{p}$ which is too large will saturate the controller output since it has a strong, direct impact on the peak of the feeding voltage. Moreover, the larger the value of $K_{p}$, the larger the overshooting will be. Another drawback induced by large values of $K_{p}$ can be seen in Fig. 6 . The motor feeding voltage has a sharp discontinuity at the beginning of each transient owing to the step input combined with a relevant proportional action. As a result, the mechanical gears and the motor are particularly stressed.

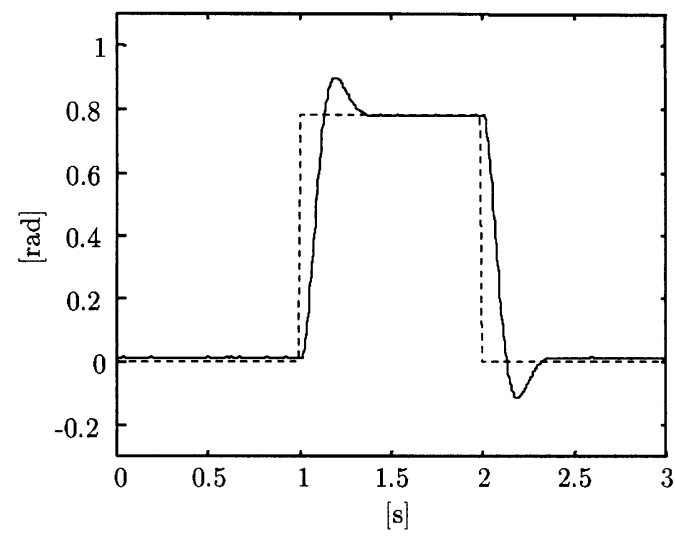

Fig. 5. Actual step response for the PD controller.

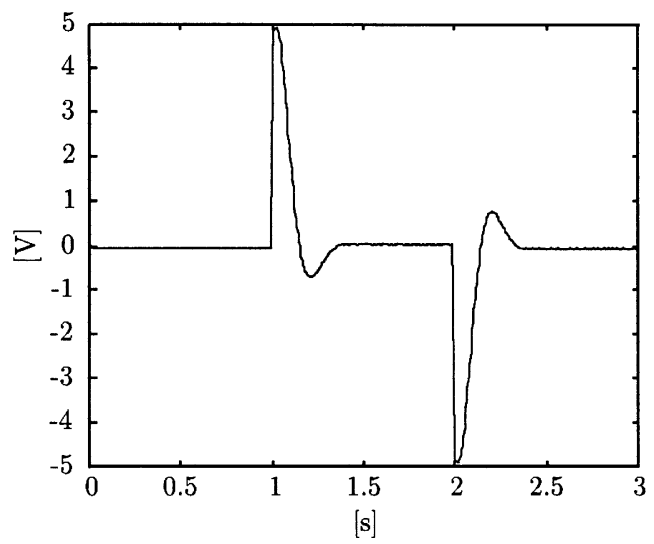

Fig. 6. Actual motor feeding voltage for the PD controller with step input.

Another relevant detail can be immediately detected from Fig. 5: the steady-state error is not zero despite the integrator term of the motor model. An average absolute steady-state error equal to $0.3867^{\circ}$ has been measured. Such an error is mainly due to the nonmodelled nonlinear friction. It is particularly relevant for the servo system considered and its influence is evident especially for low rotor velocities (static friction). The asymmetric behavior of friction is also evident: the average steady-state error depends on the direction of motion.

Still using the previously synthesized PD controller $\left(K_{p}=6.234 \mathrm{~V} / \mathrm{rad}\right.$ and $\left.K_{d}=-0.1190 \mathrm{~V} \mathrm{~s} / \mathrm{rad}\right)$, the performances of the servo system can be improved by means of the dynamic inversion approach proposed in Section 2.2. As a first step, the minimum transient time $\tau^{*}$ is evaluated for the family of output transfers $y(t ; \tau)$ defined in accordance with (13). By adopting the system parameters of Table 1 and solving the optimization problem (19), we obtained $\tau^{*}=0.2134 \mathrm{~s}$. In Fig. 7 , the resulting plot of the output signal is shown superimposed on the plot of the input command signal 
obtained via dynamic inversion. The system overshoot has been reduced but not completely suppressed. This is a consequence of the inevitable uncertainties concerning the plant parameters and of the unmodelled dynamics (nonlinear friction effects). The settling time has been slightly reduced $\left(t_{s}=0.2953 \mathrm{~s}\right)$ while a better improvement has been obtained on the average absolute steadystate error $\left(\left|e_{s}\right|=0.2613^{\circ}\right)$.

\subsection{Experimental results for the coordinated feedforwardlfeedback design using dynamic inversion}

In accordance with the new servo control system design presented in Section 3, the desired output signal is $y\left(t ; \tau^{*}\right)$. This is the optimal set-point transfer satisfying a voltage saturation avoidance constraint which has already been computed in the previous subsection $\left(\tau^{*}=0.2134 \mathrm{~s}\right)$ in agreement with (17) and (19). The coordinated feedback controller (20) is designed by first fixing $\omega_{c}=220 \mathrm{rad} / \mathrm{s}$ and then by choosing $\delta_{\text {lim }}=0.48$ for the damping ratio of the closed-loop dominant poles we infer $K_{c}=30 \mathrm{~V} / \mathrm{rad}$. Consequently, having determined

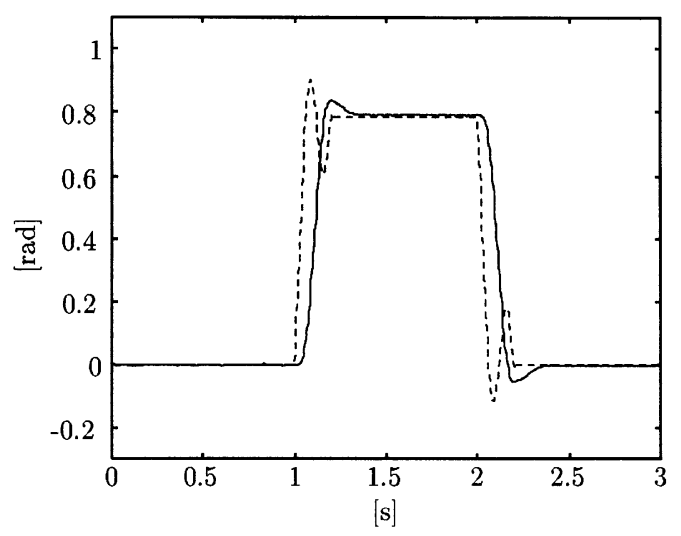

Fig. 7. Command signal (dashed line) obtained via dynamic inversion and output signal (solid line) for the closed-loop system with the PD controller. the ideal optimal output $y\left(t ; \tau^{*}\right)$ and feedback controller $G_{c}(s)$, we compute the command signal $r\left(t ; \tau^{*}\right)$ (cf. (22)) by dynamic inversion.

The experimental results are best described in Fig. 8 where the plot of the actual output signal is shown. The servo system has (almost) no overshoot and the settling time $\left(t_{s}=0.1653 \mathrm{~s}\right)$ has been greatly reduced with respect to the previously attained values (cf. Section 4.1). It is worth noting the substantial similarity between the output signal and the command signal $r\left(t ; \tau^{*}\right)$ in Fig. 8, whereas a corresponding marked difference is shown in Fig. 7 relative to the uncoordinated feedforward/feedback design. This feature of the coordinated servo scheme is both due to the controller's large bandwidth and a high loop velocity error constant $\left(K_{v}=55.88 \mathrm{~s}^{-1}\right)$. Another advantage obtained with this scheme is a sharply decreased steady-state error $\left(\left|e_{s}\right|=0.0602^{\circ}\right)$. The simulated shape of the motor supply voltage is shown in Fig. 9 compared with the shape of the actual voltage. Owing to the chosen dynamic inversion approach, $v_{\text {in }}$ is continous so that mechanical stress is reduced. As designed, the power converter never saturates.

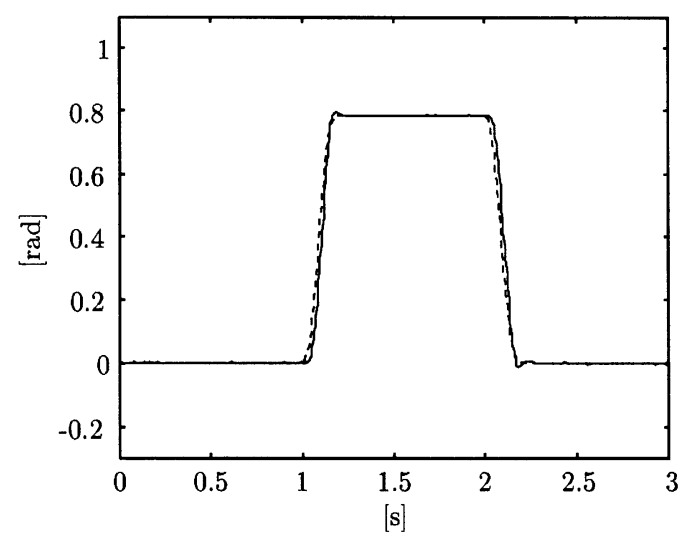

Fig. 8. Command signal (dashed line) obtained via dynamic inversion and output signal (solid line) for the new coordinated control scheme.

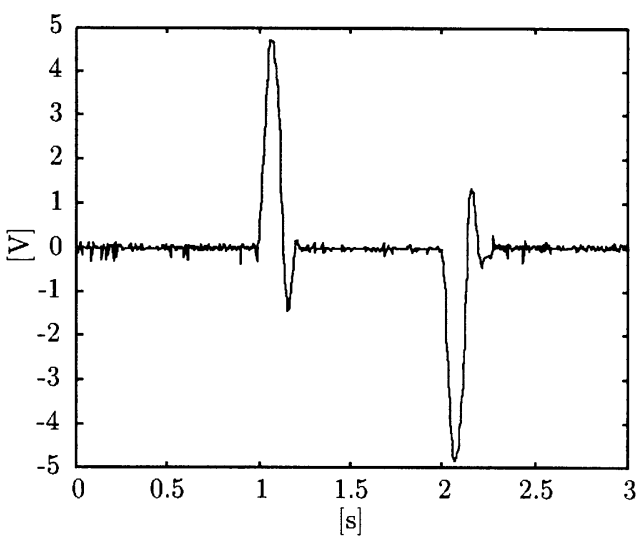

Fig. 9. Simulated and actual plot of the motor feeding voltage $v_{i n}$. 


\subsection{Experimental results with a perturbed plant}

The performances of all the three control strategies described in the paper are compared against the variation of the load inertia. More precisely, an additional arm is added to the output shaft of the dc motor (see Fig. 10). Its equivalent inertia $\left(0.982 \times 10^{-3} \mathrm{~kg} \mathrm{~m}^{2}\right)$ is about $50 \%$ of the system nominal inertia. The command signals and the controller parameters used for these experiments are the

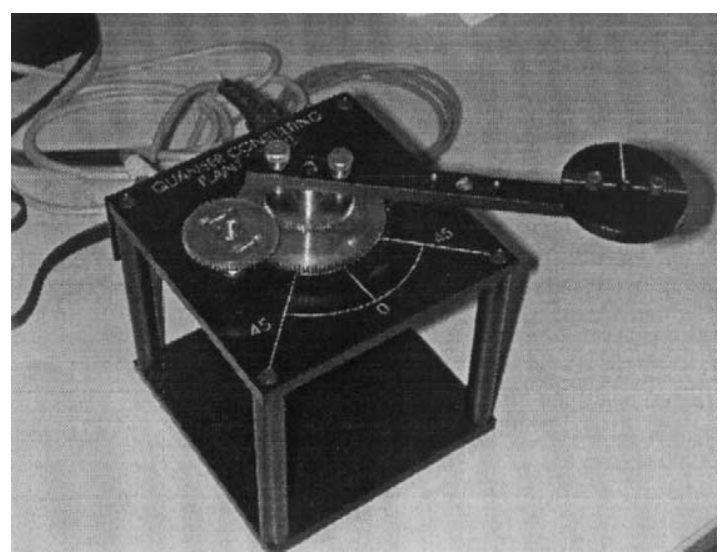

Fig. 10. The dc motor with an additional load at the output shaft.

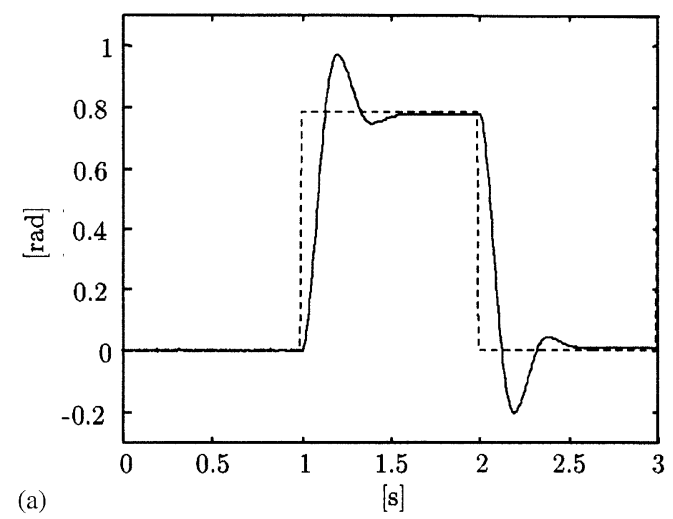

same as those used for the unperturbed plant cases. The experimental results on the perturbed plant are shown in Fig. 11. In all three experiments, both the system overshoot and the settling time are increased with respect to the values obtained for the unperturbed cases but the new inversion-based coordinated control still performs better than the control with the PD controller (comprising both the standard step input case and the inversion-based feedforward case).

Numerical results derived from all the servo experiments are shown in Table 2. Overshoots, settling times, and average absolute steady-state errors are reported for both the unperturbed and perturbed cases. These results bear out the advantages of using an inversion-based servo control scheme with a coordinated feedforward/ feedback design.

\section{Conclusions}

The basic idea of dynamic inversion is quite simple. Considering a given system, first determine a desired output signal and then, by means of an inversion-based procedure, determine the input that causes the desired output. In this paper, we exploited this idea for the servo position control of a dc motor with specifications requiring the minimizing of settling time while achieving

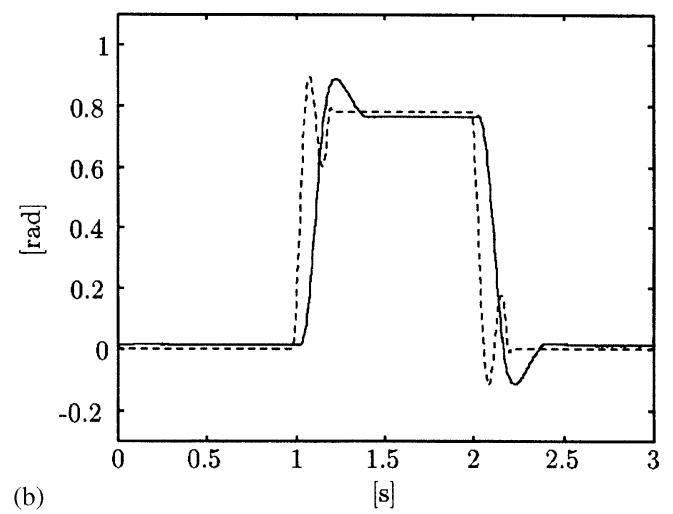

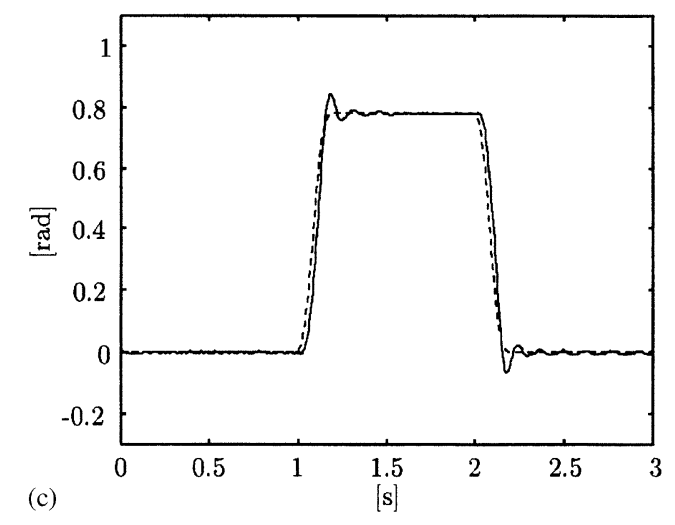

Fig. 11. Plots of the input commands and corresponding output responses for the cases: (a) standard PD controller, (b) PD controller with inversionbased feedforward, (c) new inversion-based coordinated control. 
Table 2

Comparisons of performances on the original plant and on the perturbed plant.

\begin{tabular}{|c|c|c|c|c|c|c|}
\hline & \multicolumn{2}{|c|}{ Overshoot $(\%)$} & \multicolumn{2}{|l|}{$t_{s}(\mathrm{~s})$} & \multicolumn{2}{|l|}{$\left|e_{s}\right|$ (deg.) } \\
\hline & (Original) & (Perturbed) & (Original) & (Perturbed) & (Original) & (Perturbed) \\
\hline Case (a) & 14.5 & 23.8 & 0.310 & 0.520 & 0.3867 & 0.4583 \\
\hline Case (b) & 6.5 & 13.9 & 0.295 & 0.350 & 0.2613 & 0.6990 \\
\hline Case (c) & 1.4 & 8.3 & 0.165 & 0.270 & 0.0602 & 0.1948 \\
\hline
\end{tabular}

The cases refer to: (a) standard PD controller, (b) PD controller with inversion-based feedforward, and (c) new inversion-based coordinated control.

(if possible) zero overshooting, (if possible) zero steadystate error, and a voltage saturation avoidance. Using transition polynomials (Piazzi \& Visioli, 2001b) for the output planning, two servo designs have been presented:

- a feedforward design for a traditional PD controller scheme;

- a new coordinated feedforward/feedback design using a high-gain controller.

The experimental results included in the article show how the coordinated design - for the unperturbed or original plant - outperforms the uncoordinated design with at least a $44 \%$ reduction of settling time and negligible overshooting and steady-state error. Considering an artificially perturbed plant (a 50\% increased load inertia), the coordinated design still performs better than the uncoordinated design from the standpoint of overshooting, settling time, and steady-state error (see Table 2).

Pursuing more general set-point regulation problems, inversion-based coordinated feedforward/feedback designs can be found in Piazzi and Visioli (2001c) and Piazzi and Visioli (2001a) for minimum-phase and nonminimum-phase plants, respectively.

\section{References}

Enns, D., Bugajski, D., Hendrick, R., \& Stein, G. (1994). Dynamic inversion: An evolving methodology for flight control design. International Journal of Control, 59(1), 71-91.
Guarino Lo Bianco, C., \& Piazzi, A. (2001a). A semi-infinite optimization approach to optimal spline trajectory planning of mechanical manipulators. In: M. Goberna, \& M. López (Eds.), Semi-infinite programming: Recent advances. Dordrecht, The Netherlands: Kluwer Academic Publishers, 57, 271-297.

Guarino Lo Bianco, C., \& Piazzi, A. (2001b). A hybrid algorithm for infinitely constrained optimization. International Journal of Systems Science, 32(1), 91-102.

Horowitz, I. (1963). Synthesis of feedback systems. New York: Academic Press.

Kuo, B. (1995). Automatic control systems (7th ed.). Englewood Cliffs, NJ: Prentice-Hall.

Meyer, G., Hunt, L., \& Su, R. (1995). Nonlinear system guidance, Proceedings of the 34th IEEE conference on decision and control, New Orleans, LA (pp. 590-595).

Perez, H., \& Devasia, S. (2001). Optimal output transitions for linear systems. Proceedings of the IEEE conference on decision and control, Orlando, FL (pp. 3164-3174).

Piazzi, A., \& Visioli, A. (2000). Minimum-time system inversion based motion planning for residual vibration reduction. IEEE Transactions on Mechatronics, 5(1), 12-22.

Piazzi, A., \& Visioli, A. (2001a). Optimal inversion-based control for the set-point regulation of nonminimum-phase uncertain scalar systems. IEEE Transactions on Automatic Control, 46(10), 1654-1659.

Piazzi, A., \& Visioli, A. (2001b). Optimal noncausal set-point regulation of scalar systems. Automatica, 37(1), 121-127.

Piazzi, A., \& Visioli, A. (2001c). Robust set-point constrained regulation via dynamic inversion. International Journal of Robust and Nonlinear Control, 11(1), 1-22.

Polderman, J., \& Willems, J. (1998). Introduction to mathematical system theory. New York, NY: Springer.

Teo, K., Rehbock, V., \& Jennings, L. (1993). A new computational algorithm for functional inequality constrained optimization problems. Automatica, 29(3), 789-792. 